\title{
VIOLENCIA DERIVADA DE LA ORIENTACIÓN SEXUAL EN ADULTOS MAYORES
}

\author{
Juan Enrique Nebot-Garcia \\ Departamento de Psicología Básica, \\ Clínica y Psicobiología, Universitat Jaume I \\ Castellón, España \\ junebot@uji.es \\ Naiara Martínez-Gómez \\ Departamento de Psicología Básica, \\ Clínica y Psicobiología, Universitat Jaume I \\ Castellón, España \\ Jesús Castro-Calvo \\ Departamento de Personalidad, Evaluación y \\ Tratamientos Psicológicos, \\ Universitat de València, España \\ Beatriz Gil-Juliá \\ Departamento de Personalidad, Evaluación \\ y Tratamientos Psicológicos \\ Universitat de València, España \\ Rafael Ballester-Arnal \\ Departamento de Psicología Básica, \\ Clínica y Psicobiología, Universitat Jaume I \\ Castellón, España.
}

Fecha de Recepción: 8 Marzo 2019

Fecha de Admisión: 30 Abril 2019

\section{RESUMEN}

Introducción: Las personas con una orientación sexual alternativa a la heterosexual han sufrido y siguen sufriendo discriminación por parte de sectores de la sociedad reacios a la aceptación de la diversidad, lo que en ciertos casos se traduce en agresiones verbales o físicas. La socialización y educación heteronormativa parecen estar detrás, lo que explicaría que las personas mayores (es decir, las que vivieron y se educaron en un momento histórico donde la sociedad era menos tolerante con la diversidad afectivo-sexual) hayan sido victimizadas en mayor medida. Sin embargo, la violencia hacia este colectivo permanece todavía silente y apenas disponemos de estadísticas que ofrezcan una imagen fidedigna de esta realidad. 


\section{VIOLENCIA DERIVADA DE LA ORIENTACIÓN SEXUAL EN ADULTOS MAYORES}

Objetivo: El objetivo de este trabajo es conocer qué porcentaje de personas mayores ha sufrido violencia debido a su orientación sexual.

Metodología: 449 españoles de entre 40-70 años ( $M=47,21 ; D T=6,75)$ participaron en este estudio (51,7\% hombres; $48,3 \%$ mujeres). En cuanto a la orientación, el $90,2 \%$ se autodefinía heterosexual, el 3,8\% homosexual y el $6 \%$ bisexual. Todos ellos cumplimentaron un cuestionario ad hoc sobre orientación sexual y discriminación. En concreto, la discriminación se evaluó a través de dos preguntas: «¿Has sufrido alguna vez alguna agresión verbal debido a tu orientación sexual?» y «¿Has sufrido alguna vez alguna agresión física debido a tu orientación sexual?», ambas dicotómicas («Sír»/ «No»).

Resultados: Entre homosexuales, un $85,7 \%$ de hombres y un $33,3 \%$ de mujeres ha sufrido vioIencia verbal debido a su orientación sexual; asimismo, un 21,4\% de hombres habría sufrido además agresiones físicas, frente al $0 \%$ de mujeres homosexuales. En cuanto a las personas bisexuales, un $18,8 \%$ de hombres y un $9,1 \%$ de mujeres habría recibido violencia verbal, pero ninguno referiría agresiones físicas. Finalmente, el porcentaje de personas heterosexuales que refiere haber sufrido agresiones verbales por su orientación sexual es de apenas un 3,5\% en hombres y un 1,5\% en mujeres, y sólo un 1\% de mujeres refiere agresiones físicas. Las diferencias en el porcentaje de agresiones físicas y verbales en función de la orientación sexual alcanzan en ambos casos el nivel p $<.001$.

Conclusiones: Estos resultados confirman los importantes niveles de discriminación sufridos por las personas mayores con una orientación sexual alternativa a la heterosexual, sobre todo por los hombres homosexuales. Así, de igual modo que instituciones y asociaciones desarrollan programas para prevenir la violencia LGTBI en jóvenes, se debería abordar la victimización entre adultos mayores, lo que minimizaría el impacto psicosocial en este colectivo.

Palabras clave: agresión física; agresión verbal; personas mayores; orientación sexual

\section{ABSTRACT}

Violence derived from sexual orientation in elderly adults. Introduction: People with an alternative sexual orientation to heterosexuality have suffered and continue suffering discrimination by sectors of society reluctant to accept diversity, which in certain cases, is translated into verbal or physical aggression. Socialization and heteronormative education seem to be behind, which would explain that older people (those who lived and were educated in a historical moment where society was less tolerant of affective-sexual diversity) have been victimized largely. However, the violence towards this group remains silent and we hardly have statistics that offer a reliable image of this reality.

Objective: The objective of this work is to know what percentage of the elderly have suffered violence due to their sexual orientation.

Methodology: 449 Spaniards, between 40-70 years $(M=47.21, S D=6.75)$, participated in this study ( $51.7 \%$ men, $48.3 \%$ women). In terms of orientation, $90.2 \%$ self-defined as heterosexual, $3.8 \%$ homosexual and $6 \%$ bisexual. They all completed an ad hoc questionnaire of sexual orientation and discrimination. Specifically, the discrimination was assessed through two questions: "Have you ever suffered any verbal aggression due to your sexual orientation?" and "Have you ever suffered any physical aggression due to your sexual orientation?", both dichotomous ("Yes" / "No").

Results: Among homosexuals, $85.7 \%$ of men and $33.3 \%$ of women have suffered verbal violence due to their sexual orientation; likewise, $21.4 \%$ of men have suffered physical assaults, compared to $0 \%$ of homosexual women. Regarding bisexual people, $18.8 \%$ of men and $9.1 \%$ of women have received verbal violence, but none would report physical aggression. Finally, the percentage of 
heterosexual people who reported having suffered verbal aggressions due to their sexual orientation is only $3.5 \%$ in men and $1.5 \%$ in women, and only $1 \%$ of women report physical aggression. The differences in the percentage of physical and verbal aggressions according to sexual orientation reach in both cases the level $p<.001$.

Conclusions: These results confirm the significant levels of discrimination suffered by the elderly with an alternative sexual orientation to heterosexuality, especially by homosexual men. Thus, like institutions and associations develop programs to prevent LGTBI violence in young people, victimization among older adults should be addressed too, which would minimize their psychosocial impact on this group.

Keywords: physical aggression; verbal aggression; elderly; sexual orientation

\section{INTRODUCCIÓN}

Vivimos en una sociedad heteronormativa, donde lo natural y lo normalizado son las relaciones entre un hombre y una mujer (Herz \& Johansson, 2015), y estas creencias están tan extendidas que llegan hasta el ámbito sanitario (Enson, 2015; Hayman, Wilkes, Halcomb \& Jackson, 2013; Morrison \& Dinkel, 2012), educativo (DePalma \& Jennett, 2010; McNeill, 2013; Röndahl, 2011) e incluso legislativo (International Lesbian, Gay, Bisexual, Trans and Intersex Association, 2019). Es por eso que, las personas con unas prácticas u orientaciones sexuales que se alejan de esta norma, muchas veces, deben enfrentarse a situaciones de discriminación (Digoix et al., 2016). Tal es así que, en 2008, en España, el 17\% de la población consideraba que la homosexualidad no estaba justificada, y el $5 \%$ no querría tener a una pareja de homosexuales como vecinos (Kuyper, ledema \& Keuzenkamp, 2013).

Estos prejuicios a veces llegan a materializarse en actos contra las personas LGTBI (lesbianas, gays, transexuales, bisexuales e intersexuales), de hecho, un 49,2\% de las personas LGTBI ha recibido insultos y un $13,1 \%$ ha sido agredida físicamente debido a su orientación sexual (Herek, 2009). Como consecuencia, el colectivo LGTBI suele sufrir de peor salud mental, mostrando mayores niveles de depresión (Carroll, 2015; Marchueta, 2014; Martxueta \& Etxeberria, 2014), ansiedad (Marchueta, 2014; Martxueta \& Etxeberria, 2014), e ideación suicida (Carroll, 2015; Reisner, Biello, Perry, Gamarel \& Mimiaga, 2014).

Si nos centramos en población mayor, vemos que las cifras de discriminación aumentan, siendo un $63 \%$ de personas LGTBI las que han padecido violencia verbal y un $29 \%$ las que han sufrido violencia física (D’Augelli \& Grossman, 2001). Este incremento en la discriminación sufrida puede deberse a la época en la que se socializaron y se educaron las personas mayores, un momento histórico donde la sociedad era mucho menos tolerante con la diversidad afectivo-sexual (Kuyper et al., 2013; Smith, Son \& Kim, 2014).

Pese a la importancia de la temática, tal y como ocurre con la sexualidad de las personas mayores (Cerquera, Galvis \& Cala, 2012; Freixas \& Luque, 2009), la vivencia de la propia orientación sexual en esta etapa vital es un tema escasamente estudiado (Fredriksen-Goldsen \& Kim, 2015), por lo que queda patente la necesidad de dar visibilidad a la realidad que viven las personas mayores respecto a su orientación sexual y a la discriminación recibida. Para ello, este trabajo pretende conocer qué porcentaje de personas mayores ha sufrido violencia debido a su orientación sexual y analizar si existen diferencias en las prevalencias en función de la orientación sexual, el sexo y la edad.

En cuanto a las hipótesis, se espera que: 1) En función de la orientación sexual, existirán diferencias significativas en el porcentaje de personas que han sufrido agresiones verbales y/o físicas debido a su orientación sexual, siendo las personas homosexuales las que más lo refieran. 2) En función del sexo, existirán diferencias significativas en el porcentaje de personas que han sufrido 


\section{VIOLENCIA DERIVADA DE LA ORIENTACIÓN SEXUAL EN ADULTOS MAYORES}

agresiones verbales y/o físicas debido a su orientación sexual, siendo los hombres quienes más lo padezcan. 3) Las personas que presenten mayores índices de violencia verbal y/o física serán de mayor edad que aquellas que no hayan sufrido violencia

\section{MÉTODO}

\section{Participantes}

A partir de un muestreo aleatorio simple, se seleccionaron un total de 449 españoles de entre 40 y 70 años $(M=47,21 ; D . T=6,75)$, siendo el $51,7 \%$ hombres y el $48,3 \%$ mujeres. En cuanto a la orientación sexual, el $90,2 \%$ se autodefinía como heterosexual, el 3,8\% como homosexual y el 6\% como bisexual. Los criterios de inclusión eran: tener entre 40 y 70 años y residir en España.

\section{Instrumentos de evaluación}

Además de una serie de preguntas sociodemográficas, todos los participantes cumplimentaron un cuestionario ad hoc, realizado por nuestro grupo Salusex-Unisexsida, sobre orientación sexual y discriminación. Para esta investigación en concreto, se utilizaron dos ítems para evaluar la violencia recibida a causa de la propia orientación sexual: «¿Has sufrido alguna vez alguna agresión verbal debido a tu orientación sexual?» y «¿Has sufrido alguna vez alguna agresión física debido a tu orientación sexual?». Ambas eran dicotómicas y debían responderse con «Sí» 0 «No».

\section{Procedimiento}

Como paso inicial de esta investigación, se creó un cuestionario ad hoc sobre la vivencia de la orientación sexual, realizando también una versión on-line. Para llegar a la población objetivo, durante los meses de enero a abril de 2017, se hizo difusión del estudio mediante redes sociales y foros de interés. Una vez accedían al cuestionario on-line desde sus dispositivos, se les informaba del carácter de la investigación y se les pedía el consentimiento informado. La participación era anónima, voluntaria y confidencial.

\section{Análisis estadísticos}

En primer lugar, con el programa estadístico SPSS versión 25, se realizaron análisis descriptivos de las principales variables sociodemográficas. Del mismo modo, para analizar las prevalencias de violencia sufrida y las diferencias en función de la orientación sexual y del sexo, se realizaron tablas de contingencias y pruebas de Chi cuadrado; y para las diferencias en la edad media, se utilizó la t de Student.

\section{RESULTADOS}

Un $76,5 \%$ de homosexuales y un $14,8 \%$ de bisexuales han sufrido violencia verbal debido a su orientación sexual, frente al 2,5\% de heterosexuales $\left(\chi^{2}=162,02 ; p \leq, 001\right)$. En cuanto a las agresiones físicas, un $17,6 \%$ de homosexuales y un $0,5 \%$ de heterosexuales refieren haber sufrido violencia física debido a su orientación, en cambio ninguna persona bisexual informa de haber padecido este tipo de agresión $\left(\chi^{2}=43,91 ; p \leq, 001\right)$.

En función del sexo, un 9,5\% de hombres y un 2,3\% de mujeres refieren haber sufrido violencia verbal debido a su orientación sexual $\left(\chi^{2}=10,22 ; p=, 001\right)$, frente al $1,3 \%$ de hombres y al $0,9 \%$ de mujeres que han padecido agresiones físicas $\left(\chi^{2}=0,14 ; p=, 708\right)$.

Si atendemos a las diferentes prevalencias en función del sexo y de la orientación sexual, en la Figura 1 podemos observar el porcentaje de agresiones verbales padecidas y en la Figura 2, el de agresiones físicas. 
Figura 1. Porcentaje de hombres y mujeres, con diferentes orientaciones sexuales, que refieren haber sufrido violencia verbal debido a su orientación.

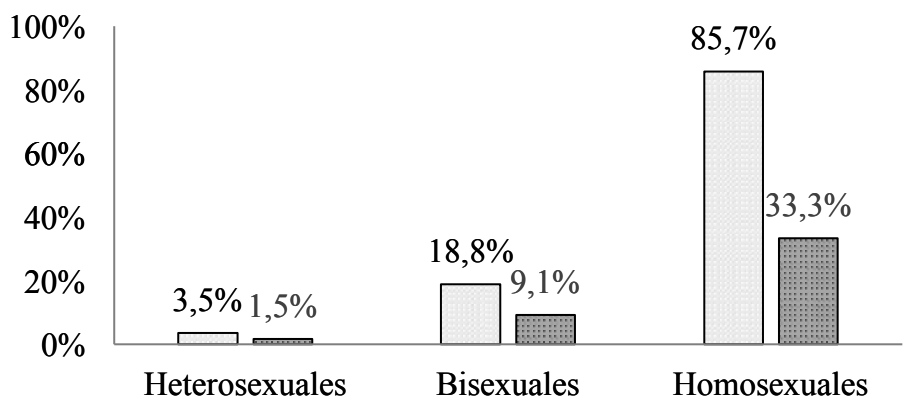

$\square$ Hombres $\square$ Mujeres

Figura 2. Porcentaje de hombres y mujeres, con diferentes orientaciones sexuales, que refieren haber sufrido violencia física debido a su orientación.

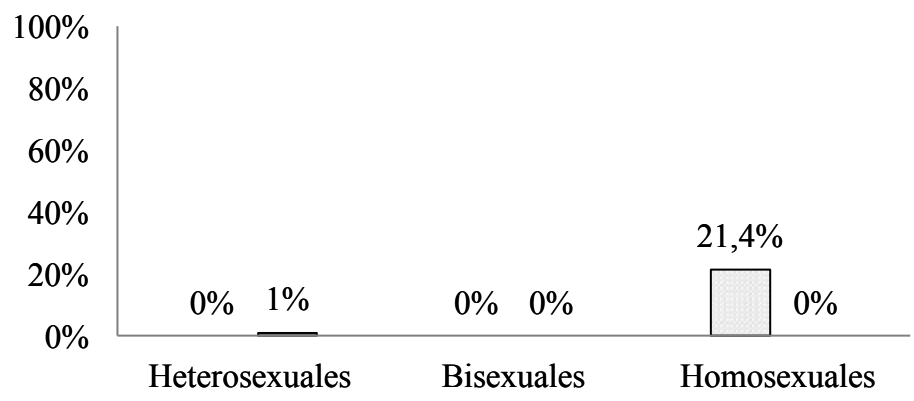

$\square$ Hombres $\square$ Mujeres

En cuanto a la variable edad, en la muestra total no se observaron diferencias significativas de edad entre aquellas personas que habían referido violencia de las que no, ni en el ámbito verbal $(t=1,11 ; p=0,267)$ ni en el físico ( $t=0,53 ; p=0,593)$. No obstante, si tenemos en cuenta a hombres y mujeres por separado (véase tabla 1), se observan diferencias significativas entre los hombres que refieren haber sufrido violencia verbal de los que no, siendo más jóvenes aquellos que la han padecido. 


\section{VIOLENCIA DERIVADA DE LA ORIENTACIÓN SEXUAL EN ADULTOS MAYORES}

Tabla 1.

Diferencias en la edad media de aquellos hombres y mujeres que refieren haber sufrido violencia (verbal y física) y aquellos que no.

\begin{tabular}{rcccc} 
& \multicolumn{2}{c}{ MUJERES } & \multicolumn{2}{c}{ HOMBRES } \\
\cline { 2 - 5 } & $\mathrm{M}(\mathrm{DT})$ & $\mathrm{t}(\mathrm{p})$ & $\mathrm{M}(\mathrm{DT})$ & $\mathrm{t}(\mathrm{p})$ \\
\hline Violencia verbal & & & & \\
Sufrida & $49,20(9,09)$ & $-1,17$ & $45,05(4,22)$ & 3,52 \\
No sufrida & $45,94(6,07)$ & $(\mathrm{p}=0,243)$ & $48,68(7,26)$ & $(\mathrm{p}=0,001)$ \\
Violencia física & & & & \\
Sufrida & $41,50(0,70)$ & 1,04 & $48,33(7,63)$ & 0,00 \\
No sufrida & $46,06(6,16)$ & $(\mathrm{p}=0,298)$ & $48,33(7,11)$ & $(\mathrm{p}=1,000)$
\end{tabular}

\section{CONCLUSIONES}

El objetivo del presente trabajo era conocer las prevalencias de violencia sufrida, debido a la propia orientación sexual, en personas mayores. Además, se pretendía analizar si existían diferencias en las prevalencias en función de la orientación sexual, el sexo y la edad.

Según los resultados, se evidencian importantes niveles de discriminación sufridos por las personas mayores con una orientación sexual alternativa a la heterosexual, siendo la homosexualidad la más castigada, tanto a nivel verbal como físico, tal y como se esperaba en la primera hipótesis. Por lo que respecta a la segunda hipótesis, ésta se cumple parcialmente, ya que a nivel físico no se encontraron diferencias significativas entre hombres y mujeres, posiblemente debido al bajo tamaño muestral en esta variable.

Así pues, nuestros resultados muestran que las personas con una orientación alternativa a la heterosexual reciben mayor discriminación (Nebot-García, Giménez-García, Ruíz-Palomino, Campos \& Ballester-Arnal, 2018), siendo los hombres homosexuales los que más violencia sufren, tanto verbal como física, lo que iría en línea con otros estudios (Herek, 2009). Este prejuicio podría deberse a la rigidez de los roles tradicionales de género (Carnaghi, Maass, \& Fasoli, 2011; Cornejo, 2015; Parrott, 2009; Parrott \& Zeichner, 2008), donde se espera que los hombres tengan unos rasgos y unas actitudes marcadamente masculinas, y las mujeres, femeninas. Los hombres heterosexuales perciben a los hombres homosexuales, a los que se les considera más femeninos, como una amenaza para la masculinidad que creen inherente en los hombres (Carnaghi et al., 2011; Glick, Gangl, Gibb, Klumpner \& Weinberg, 2007), lo que podría provocar que aparezca rechazo de la femineidad, así como emociones negativas y conductas agresivas hacia los hombres homosexuales (Cornejo, 2015; Glick et al., 2007; Parrott, 2009; Parrott \& Zeichner, 2008). No obstante, no ocurre lo mismo con la homosexualidad femenina. Los hombres heterosexuales muestran mayor aceptación de las conductas homosexuales en mujeres (Petersen \& Hyde, 2011; Poteat \& Anderson, 2012), lo que podría explicarse por la objetización sexual de la mujer, viendo la homosexualidad femenina como el cumplimiento de una fantasía sexual (Yost \& McCarthy, 2012).

En cuanto a las personas bisexuales, pese a ser un colectivo que también puede presentar conductas y experiencias sexuales con gente del mismo sexo, se observa que refieren haber sufrido menos violencia, en comparación con sus pares homosexuales. Estos hallazgos podrían ser debidos al fenómeno que se conoce como passing (Lingel, 2009; Maliepaard, 2017), que sería la capa- 
cidad de las personas bisexuales de poder pasar por heterosexuales a los ojos de la sociedad, ocultando aquellas conductas homosexuales y mostrando sólo aquellas que realizan con gente del sexo opuesto. De esta forma, las personas bisexuales se aseguran de seguir disfrutando de los privilegios que tienen las personas heterosexuales en una sociedad heteronormativa, aunque se arriesgan a que su realidad quede invisibilizada.

Por lo que respecta a las personas heterosexuales, pese a presentar bajos porcentajes de violencia recibida, sorprende que estas agresiones que refieren sean debidas a la propia orientación sexual (tal y como se especifica en la pregunta), puesto que es la normalizada en nuestra sociedad (Herz \& Johansson, 2015). En el estudio de Glick et al. (2007) se observaron emociones más negativas hacia los gays que tenían una expresión femenina, en comparación con aquellos que eran masculinos, por lo que habría que determinar que las agresiones que reciben las personas heterosexuales no se deban a otras circunstancias, como pueden ser el hecho de tener una expresión de género diferente a la esperada. Otro factor que también podría estar influyendo sería el de ser mujer. En una sociedad machista como en la que vivimos (Cepeda, 2018), el papel sexual de la mujer ha quedado, tradicionalmente, silenciado y en un segundo plano (Van Ness, Miller, Negash \& Morgan, 2017), limitándose a ser sujeto pasivo ante los deseos sexuales del hombre. Por tanto, el hecho de que una mujer presente un gran interés sexual y sea parte activa en la búsqueda de su propio placer sexual podría estar mal visto en algunos sectores de la sociedad (Baumeister \& Twenge, 2002; Emmerink, Vanwesenbeeck, van den Eijnden \& ter Bogt, 2016; Freixas \& Luque, 2009), lo que podría llegar a desencadenar insultos 0 , incluso, agresiones físicas.

Por último, en función de la edad, no se observan diferencias entre aquellas personas que refieren haber sufrido violencia debido a su orientación sexual y aquellas que no, por lo que no se cumpliría la tercera hipótesis. Del mismo modo que con la hipótesis dos, esta ausencia de significación podría deberse al bajo tamaño de la muestra en estas variables. No obstante, teniendo en cuenta el sexo de los participantes, sí que se observaron diferencias significativas entre los hombres que habían sufrido violencia verbal de aquellos que no, siendo más jóvenes los que la habían padecido. Estas diferencias podrían guardar relación con el hecho de que la gente de mayor edad suele ocultar, en mayor medida, su orientación sexual (Fredriksen-Goldsen, 2011), posiblemente porque han vivido en una sociedad más represiva (Kuyper et al., 2013; Smith et al., 2014) y tienen mayor miedo a poder ser castigados por ello. Esta ocultación de la orientación sexual haría que fueran menos visibles a los ojos de la sociedad y, por tanto, que recibieran menos discriminación que aquellas personas más jóvenes que sí que muestran su orientación sexual.

En términos generales, tanto hombres como mujeres muestran los mismos índices de aceptación de la homosexualidad femenina. En cuanto a la homosexualidad masculina, las mujeres muestran la misma tolerancia que con la femenina, en cambio, los hombres muestran mayor rechazo (Petersen \& Hyde, 2011; Poteat \& Anderson, 2012). Por ello, sería interesante, para futuras investigaciones, analizar el sexo de las personas que ejercieron dichas agresiones, físicas y verbales, para observar si existen patrones diferentes en función del sexo.

La presente investigación aporta información relevante sobre la vivencia de la orientación sexual en personas mayores, un colectivo que no siempre ha recibido la atención que merece (FredriksenGoldsen \& Kim, 2015). No obstante, hay que tener en cuenta las limitaciones que presenta el estudio, como el modesto tamaño muestral, que sería adecuado aumentar para poder obtener resultados más concluyentes, ya que en algunas variables el porcentaje de respuestas afirmativas era bajo, dificultando la posibilidad de hacer comparaciones representativas.

Con todo ello, cabría tener en cuenta todos estos resultados para desarrollar programas de prevención de la violencia contra el colectivo LGTBI, así como elaborar programas de atención a las víc- 


\section{VIOLENCIA DERIVADA DE LA ORIENTACIÓN SEXUAL EN ADULTOS MAYORES}

timas de estas agresiones, enfocándose especialmente en la gente mayor, que ha vivido en una época más represiva y menos tolerante con la diversidad afectivosexual (Kuyper et al., 2013; Smith et al., 2014), lo cual puede haber tenido, si cabe un mayor impacto psicosocial.

\section{REFERENCIAS BIBLIOGRÁFICAS}

Baumeister, R. F., \& Twenge, J. M. (2002). Cultural suppression of female sexuality. Review of General Psychology, 6(2), 166-203.

Carnaghi, A., Maass, A., \& Fasoli, F. (2011). Enhancing masculinity by slandering homosexuals: The role of homophobic epithets in heterosexual gender identity. Personality and Social Psychology Bulletin, 37(12), 1655-1665.

Carroll, J. L. (2015). Sexuality now: Embracing diversity (5th edition). EE UU: Cengage Learning.

Cepeda, I. (2018). Fighting Prejudice: Campaigns on Gender Violence in Spain. Journal of International Women's Studies, 19(6), 17-34.

Cerquera, A. M., Galvis, M. J., \& Cala, M. L. (2012). Amor, sexualidad e inicio de nuevas relaciones en la vejez: percepción de tres grupos etarios. Psychologia. Avances de la disciplina, 6(2), 7381.

Cornejo, J. (2015). Componentes ideológicos de la homofobia. Límite. Revista de Filosofía y Psicología, 7(26), 85-106.

D’Augelli, A. R. \& Grossman, A. H. (2001). Disclosure of sexual orientation, victimization, and mental health among lesbian, gay, and bisexual older adults. Journal of Interpersonal Violence, 16(10), 1008-1027.

DePalma, R. \& Jennett, M. (2010). Homophobia, transphobia and culture: Deconstructing heteronormativity in English primary schools. Intercultural Education, 21(1), 15-26.

Digoix, M., Franchi, M., Pichardo Galán, J. I., Selmi, G., de Stéfano Barbero, M., Thibeaud, M. \& Vela, J. A. M. (2016). Sexual orientation, family and kinship in France, Iceland, Italy and Spain. Families and Societies Working Paper 54(2016). Recuperado el 13 Mayo, 2017, de: http://www.familiesandsocieties.eu/wp-content/uploads/2016/08/WP54Digoixetal2016.pdf

Emmerink, P. M., Vanwesenbeeck, I., van den Eijnden, R. J., \& ter Bogt, T. F. (2016). Psychosexual correlates of sexual double standard endorsement in adolescent sexuality. The Journal of Sex Research, 53(3), 286-297.

Enson, S. (2015). Causes and consequences of heteronormativity in healthcare and education. British Journal of School Nursing, 10(2), 73-78.

Freixas, A. \& Luque, B. (2009). El secreto mejor guardado: la sexualidad de las mujeres mayores. Política y Sociedad, 46(1-2), 191-203.

Fredriksen-Goldsen, K. I. (2011). Resilience and disparities among lesbian, gay, bisexual, and transgender older adults. The Public Policy and Aging Report, 21(3), 3-7.

Fredriksen-Goldsen, K. I. \& Kim, H. J. (2015). Count me in: Response to sexual orientation measures among older adults. Research on Aging, 37(5), 464-480.

Glick, P., Gangl, C., Gibb, S., Klumpner, S., \& Weinberg, E. (2007). Defensive reactions to masculinity threat: More negative affect toward effeminate (but not masculine) gay men. Sex Roles, 57(12), 55-59.

Hayman, B., Wilkes, L., Halcomb, E. \& Jackson, D. (2013). Marginalised mothers: Lesbian women negotiating heteronormative healthcare services. Contemporary Nurse, 44(1), 120-127.

Herek, G. M. (2009). Hate crimes and stigma-related experiences among sexual minority adults in the United States: Prevalence estimates from a national probability sample. Journal of Interpersonal Violence, 24(1), 54-74. 
Herz, M. \& Johansson, T. (2015). The normativity of the concept of heteronormativity. Journal of Homosexuality, 62(8), 1009-1020.

International Lesbian, Gay, Bisexual, Trans and Intersex Association (2019). State-Sponsored Homophobia 2019. Recuperado el 1 abril, 2019, de: https://ilga.org/downloads/ILGA State_Sponsored_Homophobia_2019.pdf

Kuyper, L., ledema, J. \& Keuzenkamp, S. (2013). Towards tolerance. Exploring changes and explaining differences in attitudes towards homosexuality in Europe. The Hague: The Netherlands Institute for Social Research.

Lingel, J. (2009). Adjusting the borders: Bisexual passing and queer theory. Journal of Bisexuality, 9(3-4), 381-405.

Maliepaard, E. (2017). Bisexuality in the Netherlands: Connecting bisexual passing, communities, and identities. Journal of Bisexuality, 17(3), 325-348.

Marchueta, A. (2014). Consecuencias del bullying homofóbico retrospectivo y los factores psicosociales en el bienestar psicológico de sujetos LGB. Revista de Investigación Educativa, 32(1), 255-271.

Martxueta, A. \& Etxeberria, J. (2014). Análisis diferencial retrospectivo de las variables de salud mental en lesbianas, gais y bisexuales (LGB) víctimas de bullying homofóbico en la escuela. Revista de Psicopatología y Psicología Clínica, 19(1), 23-35.

McNeill, T. (2013). Sex education and the promotion of heteronormativity. Sexualities, 16(7), 826846.

Morrison, S. \& Dinkel, S. (2012). Heterosexism and health care: A concept analysis. Nursing Forum, $47(2), 123-130$.

Nebot-García, J. E., Giménez-García, C., Ruíz-Palomino, E., Campos, P. I., \& Ballester-Arnal, R. (2018). Malestar emocional asociado a la orientación sexual en personas mayores. International Journal of Developmental and Educational Psychology. Revista INFAD de Psicología, 4(1), 4956.

Parrott, D. J. (2009). Aggression toward gay men as gender role enforcement: Effects of male role norms, sexual prejudice, and masculine gender role stress. Journal of Personality, 77(4), 11371166.

Parrott, D. J., \& Zeichner, A. (2008). Determinants of anger and physical aggression based on sexual orientation: An experimental examination of hypermasculinity and exposure to male gender role violations. Archives of Sexual Behavior, 37(6), 891-901.

Petersen, J. L., \& Hyde, J. S. (2011). Gender differences in sexual attitudes and behaviors: A review of meta-analytic results and large datasets. Journal of Sex Research, 48(2-3), 149-165.

Poteat, V. P., \& Anderson, C. J. (2012). Developmental changes in sexual prejudice from early to late adolescence: The effects of gender, race, and ideology on different patterns of change. Developmental Psychology, 48(5), 1403-1415.

Reisner, S. L., Biello, K., Perry, N. S., Gamarel, K. E. \& Mimiaga, M. J. (2014). A compensatory model of risk and resilience applied to adolescent sexual orientation disparities in non-suicidal self-injury and suicide attempts. American Journal of Orthopsychiatry, 84(5), 545-546.

Röndahl, G. (2011). Heteronormativity in health care education programs. Nurse Education Today, 31(4), 345-349.

Smith, T. W., Son, J. \& Kim, J. (2014). Public attitudes toward homosexuality and gay rights across time and countries. NORC at the University of Chicago and the Williams Institute. Recuperado el 25 marzo, 2019, de: http://williamsinstitute.law.ucla.edu/wp-content/uploads/public-attitudesnov-2014.pdf 
Van Ness, N., Miller, M. M., Negash, S., \& Morgan, M. (2017). Embracing our eroticism: a foucauldian discourse analysis of women's eroticism. Journal of Feminist Family Therapy, 29(3), 103-126.

Yost, M. R., \& McCarthy, L. (2012). Girls gone wild? Heterosexual women's same-sex encounters at college parties. Psychology of Women Quarterly, 36(1), 7-24. 